\title{
Mixobiota do Parque Nacional Serra de Itabaiana, SE, Brasil: Physarales
}

\author{
Maria de Fátima de Andrade Bezerra ${ }^{1}$, Andrea Carla Caldas Bezerra ${ }^{2}$, \\ Alissandra Trajano Nunes ${ }^{3}$, Carlos Lado ${ }^{4}$ e Laise de Holanda Cavalcanti ${ }^{3,5}$
}

Recebido em 5/04/2007. Aceito em 27/02/2008

\begin{abstract}
RESUMO - (Mixobiota do Parque Nacional Serra de Itabaiana, Sergipe, Brasil: Physarales). Visando contribuir com informações sobre a diversidade de Myxomycetes em florestas úmidas e savanas Neotropicais, foram inventariadas as espécies de Physarales associadas a diferentes microhabitats no Parque Nacional Serra de Itabaiana, Sergipe, Brasil (1040'52'’S e 37²5'15'W, $180-670$ m, 7966 ha). Durante 20 meses consecutivos foram efetuadas coletas de esporocarpos e de amostras de vários tipos de substratos a serem utilizados na preparação de 590 câmaras-úmidas. Coleções representativas do material estudado foram depositadas no Herbário UFP, com duplicatas no herbário MA - Fungi. A abundância e a constância de cada espécie foram determinadas. Apresenta-se uma lista comentada das sete espécies de Didymiaceae e 24 espécies de Physaraceae identificadas e sua distribuição no Brasil. As Physarales estão presentes em todos os microhabitats analisados, esporulando na estiagem e na estação chuvosa; fimícolas e suculentícolas foram registradas apenas em câmara-úmida. As espécies mais abundantes foram Physarum viride (Bull.) Pers., P. stellatum (Massee) G.W. Martin e P. cinereum (Batsch) Pers. Exceto Diachea silvaepluvialis M.L. Farr, P. cinereum e Physarum roseum Berk. \& Broome, todos os táxons constituem primeira referência para Sergipe. Diderma rugosum (Rex) T. Macbr., Didymium dubium Rostaf. e Physarum pulcherrimum Berk. \& Ravenel estão sendo referidas pela primeira vez para o Brasil.
\end{abstract}

Palavras-chave: Cerrado, Floresta Atlântica, Myxomycetes, Neotrópicos, Physarales

\begin{abstract}
Myxobiota of Serra de Itabaiana National Park, Sergipe State, Brazil: Physarales). The aims of this study were to obtain data on Myxomycete diversity in Neotropical forests and savannas and to determine the composition of the Physarales species assemblage associated with different microhabitats in Serra de Itabaiana National Park, Sergipe (1040'52' S; 37² $25^{\prime} 15^{\prime}$ ' W, 180-670m alt., 7966 ha). During 20 months, sporocarps were collected and samples of substrata obtained for use in 590 moist-chamber cultures. Collections are deposited in the Herbaria UFP and MA - Fungi. The abundance and constancy of each species are given. Seven species of Didymiaceae and 24 species of Physaraceae comprise an annotated list and their distribution in Brazil is presented. Physarales were present in all microhabitats, in the dry and rainy seasons; fimicolous and succulenticolous species were observed only in moist-chamber cultures. The most abundant species were Physarum viride (Bull.) Pers., Physarum stellatum (Massee) G.W. Martin, and Physarum cinereum (Batsch) Pers. Except for Diachea silvaepluvialis M.L. Farr, P. cinereum, and Physarum roseum Berk. \& Broome, all the species are new records for Sergipe. Diderma rugosum (Rex) T. Macbr., Didymium dubium Rostaf. and Physarum pulcherrimum Berk. \& Ravenel are reported for the first time in Brazil.
\end{abstract}

Kew words: Atlantic forest, savanna, Myxomycetes, Neotropics, Physarales

\section{Introdução}

A ordem Physarales compreende as famílias Physaraceae, com oito gêneros e 193 espécies, Didymiaceae, com sete gêneros e 169 espécies e Elaeomyxaceae, com um gênero e duas espécies (Martin et al. 1983; Lado 2001). Grande parte das espécies dessa ordem tem ampla distribuição mundial, porém algumas parecem ser restritas às regiões tropicais, como Physarum roseum Berk. \& Broome, enquanto outras habitam ambientes muito particulares, como Diderma alpinum (Meyl.) Meyl. e Diderma fallax (Rostaf.) Lado, tipicamente nivícolas, esporulando na neve fundente (Lado 2004).

Analisando-se obras como as de Lister (1925), Hagelstein (1944), Macbride \& Martin (1934), Martin \& Alexopoulos (1969), Nannenga-Bremekamp (1991), Farr (1976) e Lakhanpal \& Mukerji (1981), fica evidente que a maioria das Physaraceae esporula sobre madeira em decomposição e poucas espécies são citadas como

\footnotetext{
1 Universidade Federal de Pernambuco, Programa de Pós-Graduação em Biologia de Fungos (fatandrade@ hotmail.com)

2 Universidade Federal de Pernambuco, Programa de Pós-Graduação em Ciências Biológicas

3 Universidade Federal de Pernambuco, Departamento de Botânica, Laboratório de Myxomycetes, Av. Prof. Moraes Rego s.n., Cidade Universitária, 50670-901 Recife, PE, Brasil

4 Departamento de Micologia, Real Jardin Botánico de Madrid, Plaza de Murillo 2, E- 28014 Madrid, España (lado@ rjb.csic.es)

5 Autor para correspondência: laise@ pesquisador.cnpq.br
} 
foliícolas, comportamento mais encontrado entre as Didymiaceae. Algumas espécies de Physarales ocupam microhabitats especiais, desenvolvendo-se e esporulando sobre fezes de herbívoros, como Badhamia apiculospora (Härk.) Eliasson \& N. Lundq. (Eliasson \& Keller 1999); outras, comportam-se como micetícolas, freqüentemente associadas a Basidiomicetos, como Badhamia utricularis (Bull.) Berk. e Physarum pezizoideum (Jungh.) Pavill. \& Lagarde (Lister 1925). Várias espécies são corticícolas, estando 55 Physaraceae e 21 Didymiaceae representadas na chave de identificação elaborada por Mitchell (2004) para espécies encontradas em cascas de árvores vivas. As Physarales corticícolas são encontradas tanto em ambientes naturais quanto antropizados, sendo Physarum compressum Alb. \& Schwein., P. leucophaeum Fr. e Diderma deplanatum Fr. incluídas entre as presentes em parques e praças de grandes cidades, como Madri e Helsinki (Basanta 2004; Härkönen \& Vänskä 2004). Microhabitats só recentemente explorados para os mixomicetos também são ocupados pelos representantes dessa ordem, como Badhamia melanospora Speg., tipicamente suculentícola e $P$. compressum, freqüentemente encontrado em inflorescências de grandes ervas tropicais (Schnittler \& Stephenson 2002).

No Brasil, as Physarales estão representadas pelas famílias Physaraceae e Didymiaceae, com 11 gêneros e 79 espécies, distribuídos em todas as regiões do país, porém sem registros até o momento para vários estados (Cavalcanti 2002; Maimoni-Rodella 2002; Putzke 2002). No levantamento da mixobiota sergipana, com coletas iniciadas em 2002, a ocorrência de espécies dessa ordem foi registrada no Parque Nacional Serra de Itabaiana, em diferentes microhabitats. Visando contribuir com informações sobre a diversidade de Myxomycetes em florestas úmidas e savanas Neotropicais, comenta-se no presente trabalho a distribuição dessas espécies nos diferentes ambientes vegetacionais e microhabitats existentes no Parque Nacional Serra de Itabaiana, ampliando assim o conhecimento sobre sua distribuição mundial e no Brasil.

\section{Material e métodos}

Situado na Mesorregião do Agreste de Sergipe, Nordeste do Brasil, o Parque Nacional Serra de Itabaiana - PNSI $\left(10^{\circ} 40 ' 52\right.$ ' S e $37^{\circ} 25^{\prime} 15^{\prime \prime} \mathrm{W}$, 180-660 m, 7.966 ha) abrange os municípios de Areia Branca, Laranjeiras, Itaporanga d'Ajuda e Campo do Brito; o clima local é quente e úmido, com chuvas de outono-inverno (1.200 $\mathrm{mm}$ a $1.300 \mathrm{~mm} / \mathrm{ano}) \mathrm{e}$ temperaturas médias mensais oscilando entre $17^{\circ} \mathrm{C}$ e $23{ }^{\circ} \mathrm{C}$, oferecendo condições favoráveis ao desenvolvimento dos Myxomycetes (CONDESE 1978).
Os espécimes de Physarales (campo e câmaraúmida) foram obtidos em 51 dias de trabalhos de campo, distribuídos em 19 excursões realizadas em um período de 20 meses consecutivos, com dois a quatro dias de permanência em campo. Na vertente Leste da Serra as coletas foram efetuadas nas localidades Riacho Coqueiro (mata de galeria), Mangabeira (campo cerrado), Gruta da Serra, trilha de acesso ao Salão do Rio dos Negros e no topo da Serra (capão de mata e campo limpo); na vertente Oeste foram coletadas amostras em área de Floresta Mesófila Decídua, na localidade Bula Cinza e no Sítio Noel. Foram explorados os seguintes tipos de substrato: folhas mortas de palmeiras (Arecaceae) e bromélias (Bromeliaceae) ainda presas à planta mãe; bases foliares de canela-de-ema (Vellozia dasypus Seub., Velloziaceae) ainda presas ao caule; casca de árvores vivas, como mangabeira (Hancornia speciosa Gomes, Apocynaceae), jaqueira (Artocarpus heterophyllus Lam., Moraceae) e outras não identificadas; troncos mortos ainda em pé ou caídos; partes vegetativas de coroa de frade (Melocactus sp., Cactaceae); liter foliar (folhas, gravetos etc); fezes diurnas de coelho brasileiro (Sylvilagus brasiliensis L.) e fezes de cavalo (Equus caballus L.). Com estes mesmos substratos, provenientes das diferentes localidades encontradas no PNSI, foram preparadas 590 câmaras-úmidas, seguindo-se a metodologia descrita por Stephenson et al. (1999).

Para identificação das espécies foram empregados os trabalhos de Lister (1925), Martin \& Alexopoulos (1969) e Farr (1976), seguindo-se o sistema de classificação de Martin et al. (1983). Seguiu-se Lado (2001) na indicação dos binômios e autores das espécies. A distribuição geográfica das espécies no Brasil baseouse nos trabalhos de Cavalcanti (2002), Maimoni-Rodella (2002) e Putzke (2002).

Exsicatas representativas do material estudado encontram-se depositadas no herbário UFP (Universidade Federal de Pernambuco, Recife), com duplicatas no Herbário MA - Fungi (Departamento de Micologia, Real Jardim Botânico, Madri).

As frutificações foram classificadas como abundantes (> 30 esporocarpos), médias (30-10 esporocarpos) ou escassas (< 10 esporocarpos). A constância das espécies foi calculada relacionando-se o número de excursões nas quais a espécie foi coletada e o total de excursões realizadas (19), expressa em porcentagem (Cavalcanti \& Mobin 2004); cada espécie foi enquadrada como constante (>50\%), acessória $(25 \%$ a $50 \%)$ ou acidental $(<25 \%)$. A abundância de cada espécie foi calculada como o percentual de amostras em relação ao total obtido, adotando-se as quatro classes de Schnittler et al. (2002): escassa $(<1,5 \%)$, ocasional $(1,5-3,5 \%)$, comum $(>3,5-6,5 \%)$ ou abundante 
(>6,5\%). A semelhança entre o conjunto de espécies registradas no cerrado e na mata de galeria foi avaliada empregando-se o coeficiente de comunidade (Stephenson 1988).

\section{Resultados e discussão}

O estudo dos espécimes obtidos no PNSI (campo e câmara-úmida) revelou a presença de 24 representantes das Physaraceae, distribuídos em cinco gêneros (Badhamia, Craterium, Fuligo, Physarella, Physarum) e sete espécies de Didymiaceae, pertencentes aos gêneros Diachea, Diderma e Didymium. Com exceção de Diachea silvaepluvialis M.L. Farr, Physarum cinereum (Batsch) Pers. e Physarum roseum Berk. \& Broome, os táxons a seguir relacionados estão sendo referidos pela primeira vez para Sergipe. Diderma rugosum (Rex) T. Macbr., Didymium dubium Rostaf., Physarum aff. conglomeratum (Fr.) Rostaf. e P. pulcherrimum Berk. \& Ravenel. ainda não eram conhecidas como componentes da mixobiota brasileira.

\section{DIDYMIACEAE}

1. Diachea silvaepluvialis M.L. Farr, Contr. U.S. Natl. Herb. 37(6): 409. 1969.

Frutificação abundante, $>50$ esporângios pedicelados, sobre folhas de dicotiledôneas não identificadas. Coletada apenas uma vez no PNSI, durante a estiagem (setembro), em ambiente de mata de galeria (Riacho Coqueiro, $180 \mathrm{~m}$ alt.); os esporocarpos são muito típicos, destacando-se os esporos castanho-enegrecidos, com longos espinhos. Esta espécie só recentemente foi registrada para o Brasil, ocorrendo em áreas de floresta úmida nos Estados de Sergipe e Paraíba.

Distribuição no Brasil: Região Nordeste (Paraíba, Sergipe).

Material examinado: BRASIL. Sergipe: Areia Branca, Parque Nacional Serra de Itabaiana, 13/XI/2002, Bezerra, MFA 134 (UFP).

2. Diderma deplanatum Fr., Syst. Mycol. 3: 110. 1829.

Frutificações escassas, 1-3 plasmodiocarpos, sobre folhas de dicotiledôneas não identificadas, coletadas em mata de galeria (Riacho Coqueiro, $180 \mathrm{~m}$ alt.), durante a estiagem (setembro).

Diderma deplanatum tem ocorrência conhecida apenas em três estados, situados em diferentes regiões do país, sendo uma das poucas espécies de mixomicetos registradas para o Mato Grosso (Cavalcanti 2002).

Distribuição no Brasil: Região Nordeste (Pernambuco), Centro-Oeste (Mato Grosso) e Sudeste (São Paulo).
Material examinado: BRASIL. Sergipe: Areia Branca, Parque Nacional Serra de Itabaiana, 13/IX/2002, Bezerra, MFA 462 (UFP); idem, 13/IX /2002, Bezerra, MFA 463 (UFP).

3. Diderma effusum (Schwein.) Morgan, J. Cincinnati Soc. Nat. Hist. 16: 155. 1894.

Frutificação escassa, constituída por apenas um plasmodiocarpo, desenvolvido sobre folha morta de Bromeliaceae, coletado na vertente Leste da Serra, a $471 \mathrm{~m}$ alt., durante a estiagem (dezembro).

Distribuição no Brasil: Região Norte (Amazonas), Nordeste (Paraíba, Pernambuco), Sudeste (Espírito Santo, São Paulo), Sul (Paraná, Rio Grande do Sul).

Material examinado: BRASIL. Sergipe: Areia Branca, Parque Nacional Serra de Itabaiana, 21/XII/2003, Bezerra, MFA 692 (UFP).

4. Diderma rugosum (Rex) T. Macbr., N. Amer. SlimeMoulds: 105. 1899.

Fig. 1a

Frutificações escassas, seis a 12 esporângios pedicelados, subglobosos, 1,0-1,3 $\mathrm{mm}$ alt., esporoteca branco-acinzentada; hipotalo não visualizado; pedicelo castanho-enegrecido, brilhante, afinando levemente para o ápice, cerca de $2 / 3$ da altura total; perídio simples, cartilaginoso, fino, marcado com reticulações que the dão aspecto enrugado, deiscência pela ruptura das linhas que marcam as reticulações; capilício hialino, dicotomicamente ramificado; columela clavada, com superfície rugosa; esporo globoso, castanho-violáceo sob luz transmitida, densamente verrucoso, 7,5-9,0 $\mu \mathrm{m}$ diâm.

Sobre briófitas, em tronco morto caído e folhas, coletado no topo da Serra (654 m alt.), durante a estiagem (fevereiro, novembro). Os esporocarpos obtidos são muito típicos, tanto no que se refere às características do perídio e da columela quanto do ambiente em que se desenvolveram, onde os musgos são abundantes, como freqüentemente reportado para a espécie. Esta espécie, raramente coletada, foi referida por Farr (1976) apenas para a Flórida (USA), Dominica, Jamaica, Trindade e Antigua; recentemente foi referida para o México (EstradaTorres et al. 2001), também coletada sobre briófitas.

Distribuição no Brasil: primeiro registro para o país.

Material examinado: BRASIL. Sergipe: Areia Branca, Parque Nacional Serra de Itabaiana, 14/II/2003, Bezerra, MFA 121 (UFP); idem, 27/XI/2003, Bezerra, MFA 775a (UFP); idem, 27/XI/2003, Bezerra, MFA 776 (UFP).

\section{Diderma sp.}

Frutificação escassa, constituída por esporângios depressos, densamente superpostos de modo a formar 
uma espécie de pseudoetálio irregular, branco; hipotalo branco, brilhante, irregular, membranoso; perídio branco, calcário, duplo, que se desprende através de linhas marcadas em fragmentos poligonais; capilício castanho claro, liso, 1,5 um diâm.; esporos castanhos, subglobosos, densamente verrucosos, 7,5-9,0 $\mu \mathrm{m}$ diâm. Desenvolvido sobre briófitas, em tronco morto caído, coletado durante a estação chuvosa (agosto), na trilha de acesso ao Salão do Rio dos Negros (210 m alt.) .

$\mathrm{O}$ aspecto da frutificação é extremamente semelhante à fotografia do holótipo de Diderma acanthosporum Estrada et Lado descrita no início deste século para o México e conhecida apenas de florestas secas tropicais e subtropicais (Estrada-Torres et al. 2001). O menor diâmetro e a ornamentação dos esporos não se enquadram na descrição da espécie, assemelhando-se mais aos de D. hemisphaericum (Bull.) Hornem. (6,0-9,0 $\mu \mathrm{m})$ que, todavia, não forma frutificações pseudoetalióides.

Material examinado: BRASIL. Sergipe: Areia Branca, Parque Nacional Serra de Itabaiana, 16/VIII/2002, Bezerra, MFA 158 (UFP).

6. Didymium clavus (Alb. \& Schwein.) Rabenh., Deutschl. Krypt.-Fl. 1: 280. 1844.

Frutificações desde escassas até abundantes, seis a 35 esporângios pedicelados, esporulados sobre tronco morto caído (1) e folhas mortas (3) de Bromeliaceae, coletados na Serra a $427 \mathrm{~m}$ alt., durante a estiagem (dezembro).

Distribuição no Brasil: Região Norte (Roraima), Nordeste (Bahia, Pernambuco, Piauí), Sudeste (São Paulo) e Sul (Paraná, Rio Grande do Sul, Santa Catarina).

Material examinado: BRASIL. Sergipe: Areia Branca, Parque Nacional Serra de Itabaiana, 21/XII/2003, Bezerra, MFA 675 (UFP); idem, 21/XII/2003, Bezerra, MFA 676 (UFP); idem, 21/XII/2003, Bezerra, MFA $681 b$ (UFP); idem, 21/XII/2003, Bezerra, MFA 683 (UFP); idem, 21/XII/2003, Bezerra, MFA 684 (UFP); idem, 21/XII/2003, Bezerra, MFA 690 (UFP).

7. Didymium dubium Rostaf., Sluzowce Monogr.: 152. 1874.

Fig. 1b

Frutificação escassa, constituída por oito plasmodiocarpos, planos a achatados, isolados, brancocinza; hipotalo não visualizado; perídio simples, deiscência irregular, hialino a castanho, face interna iridescente, membranáceo, com cristais de cálcio medindo 5 a $7 \mu \mathrm{m}$ larg.; capilício castanho, liso, levemente elástico; esporos castanhos sob luz transmitida, globosos, verrucosos, (9)10-11 $\mu \mathrm{m}$ diâm.
Os esporocarpos da única amostra obtida desenvolveram-se após 20 dias em câmara-úmida preparada com folhas de canela de ema (Vellozia dasypus Seub., Velloziaceae), coletadas no Cerrado (Mangabeira, $210 \mathrm{~m}$ alt.), durante a estiagem (fevereiro).

Segundo a literatura, esta é uma espécie de zonas temperadas e inclui-se entre as nivícolas, como pode ser visto na lista apresentada recentemente por Lado (2004) e Lado et al. (2005). Para os Neotrópicos não foi incluída por Farr (1976) entre as espécies ocorrentes no Brasil, mas a autora cita sua presença na Colômbia e Venezuela, em altitudes elevadas (2.000-4.000 m).

O microhabitat onde o espécime foi coletado, constituído pelas bases foliares de canela de ema ainda presas ao tronco, provavelmente oferece um microclima favorável ao desenvolvimento de $D$. dubium nas condições de luminosidade e temperatura elevadas do cerrado, bem distintas das vigentes nos ambientes onde esta espécie costuma ser encontrada.

Distribuição no Brasil: primeiro registro para o país.

Material examinado: BRASIL. Sergipe: Areia Branca, Parque Nacional Serra de Itabaiana, 7/III/2003, Bezerra, MFA 291 (UFP).

\section{PHYSARACEAE}

\section{Badhamia sp.}

Frutificação abundante, composta por esporângios pedicelados, globosos a subglobosos, gregários; hipotalo branco-gelo a branco-amarelado, circular, membranoso; pedicelo amarelo-palha na base e castanho-amarelado no ápice, cilíndrico, contorcido, 525,5-709,5 ㅆ diâm.; perídio persistente, deiscência através de fragmentação irregular a longitudinal; pseudo-columela branca; capilício hialino, nódulos branco-gelo, irregulares; esporada castanho escuro; esporos castanhos, globosos, espinulosos, 7,0-8,0 $\mu \mathrm{m}$ diâm.

O único espécime obtido é constituído por 43 esporângios pedicelados, desenvolvidos sobre tronco morto caído, coletados na Gruta da Serra (220 m alt.), durante a estação chuvosa (junho). Os esporocarpos são macroscopicamente muito semelhantes aos de Badhamia melanospora Speg. (= B. gracilis (T. Macbr.) T. Macbr.), porém o capilício é mais delicado e os esporos, apesar de coincidirem na coloração e ornamentação, apresentam menor diâmetro $(7,5 \mu \mathrm{m})$. Nenhuma das espécies deste gênero citadas para o Brasil possui esporos menores que $8 \mu \mathrm{m}$.

Material examinado: BRASIL. Sergipe: Areia Branca, Parque Nacional Serra de Itabaiana, 19/VI/2003, Bezerra, MFA 553 (UFP).

2. Craterium aureum (Schumach.) Rostaf., Sluzowce Monogr.: 124. 1874. 
Frutificação abundante, 51 esporângios pedicelados, sobre tronco vivo com briófitas, coletados em mata de galeria (Riacho Coqueiro, $180 \mathrm{~m}$ alt.), durante a estação chuvosa (junho).

Distribuição no Brasil: Região Norte (Amazonas, Roraima), Nordeste (Paraíba, Pernambuco, Piauí).

Material examinado: BRASIL. Sergipe: Areia Branca, Parque Nacional Serra de Itabaiana, 17/VI/2003, Bezerra, MFA 488 (UFP).

3. Craterium leucocephalum (Pers. ex. J.F. Gmel.) Ditmar in Sturm, Deutschl. Fl. Pilze 1(1): 21. 1812.

Frutificações abundantes, $>30$ esporângios pedicelados, sobre folhas de Bromeliaceae, coletadas na Serra a $427 \mathrm{~m}$ alt., durante a estiagem (dezembro).

Distribuição no Brasil: Região Norte (Amazonas, Roraima), Nordeste (Bahia, Paraíba, Pernambuco), Sudeste (São Paulo), Sul (Rio Grande do Sul, Santa Catarina).

Material examinado: BRASIL. Sergipe: Areia Branca, Parque Nacional Serra de Itabaiana, 21/XII/2003, Bezerra, MFA 678 (UFP); idem, 21/XII/2003, Bezerra, MFA 679 (UFP); idem, 21/XII/2003, Bezerra, MFA 682 (UFP); idem, 21/XII/2003, Bezerra, MFA 686 (UFP); idem, 21/XII/2003, Bezerra, MFA 688 (UFP); idem, 21/XII/2003, Bezerra, MFA 689 (UFP).

4. Craterium paraguayense (Speg.) G. Lister in Lister, Monogr. Mycetozoa, ed. 2: 95. 1911.

Frutificações abundantes, $>50$ esporângios pedicelados, com um espécime coletado sobre tronco morto caído e os demais sobre folhas mortas de dicotiledôneas não identificadas, ambos em mata de galeria (Riacho Coqueiro, $180 \mathrm{~m}$ alt.), durante a estiagem (novembro, dezembro) e na estação chuvosa (julho).

Distribuição no Brasil: Região Norte (Amazonas), Nordeste (Pernambuco, Piauí), Sudeste (São Paulo).

Material examinado: BRASIL. Sergipe: Areia Branca, Parque Nacional Serra de Itabaiana, 14/VII/2002, Bezerra, MFA 107 (UFP; MA-Fungi); idem, 14/VII/2002, Bezerra, MFA 108 (UFP); idem, 13/VII/2002, Bezerra, MFA 312 (UFP); idem, 13/VII/2002, Bezerra, MFA 349 (UFP; MA-Fungi); idem, 14/VII/2002, Bezerra, MFA 350 (UFP); idem, 14/VII/2002, Bezerra, MFA 351 (UFP; MA-Fungi); idem, 18/XI/2002, Bezerra, MFA 418 (UFP; MA-Fungi); idem, 18/XI/2002, Bezerra, MFA 419 (UFP); idem, 21/XII/2003, Bezerra, MFA 678 (UFP); idem, 21/XII/2003, Bezerra, MFA 679 UFP.

5. Fuligo septica (L.) F.H. Wigg., Prim. Fl. Holsat: 112. 1780 .

Frutificação escassa constituída por dois etálios, esporulados sobre tronco morto caído na Gruta da Serra
(220 $\mathrm{m}$ alt.), durante a estiagem (março).

Distribuição no Brasil: Região Norte (Amazonas, Roraima), Nordeste (Alagoas, Bahia, Ceará, Pernambuco), Sudeste (Minas Gerais, São Paulo), Sul (Paraná, Rio Grande do Sul, Santa Catarina).

Material examinado: BRASIL. Sergipe: Areia Branca, Parque Nacional Serra de Itabaiana, 14/III/2003, Bezerra, MFA 122 (UFP).

6. Physarella oblonga (Berk. \& M.A. Curtis) Morgan, J. Cincinnati Soc. Nat. Hist. 19: 7. 1896.

Frutificações desde escassas até abundantes, constituídas por oito a $>50$ esporângios pedicelados, todas sobre troncos mortos caídos na mata de galeria (Riacho Coqueiro, $180 \mathrm{~m}$ alt.), coletadas tanto na estiagem (setembro, outubro) como na estação chuvosa (julho, agosto).

Distribuição no Brasil: Região Norte (Amazonas, Pará), Nordeste (Bahia, Paraíba, Pernambuco, Piauí, Rio Grande do Norte), Sudeste (São Paulo), Sul (Paraná, Rio Grande do Sul, Santa Catarina).

Material examinado: BRASIL. Sergipe: Areia Branca, Parque Nacional Serra de Itabaiana, 13/VIII/2002, Bezerra, MFA 106 (UFP); idem, 14/VIII/2002, Bezerra, MFA 115 (UFP); idem, 14/VII/2002, Bezerra, MFA 116 (UFP); idem, idem, 18/X/2002, Bezerra, MFA 420 (UFP); idem, 14/VIII/2002, Bezerra, MFA 652 (UFP); idem, 14/X/2003, Bezerra, MFA 733 (UFP); idem, 12/IX/2003, Bezerra, MFA 865 (UFP).

7. Physarum album (Bull.) Chevall., Fl. Gén. Env. Paris 1: 336. 1826.

Frutificações de médias até abundantes, 14 a mais de 50 esporângios pedicelados, todas coletadas sobre troncos mortos caídos, na mata de galeria (Riacho Coqueiro, $180 \mathrm{~m}$ alt.), no Alojamento do IBAMA, (210 m alt.) e na Gruta da Serra (220 m alt.), durante a estação chuvosa (junho, agosto).

Distribuição no Brasil: Região Nordeste (Bahia, Ceará, Maranhão, Paraíba, Pernambuco, Piauí), Sudeste (Espírito Santo, Minas Gerais, Rio de Janeiro, São Paulo), Sul (Paraná, Santa Catarina, Rio Grande do Sul).

Material examinado: BRASIL. Sergipe: Areia Branca, Parque Nacional Serra de Itabaiana, 18/VI/2003, Bezerra, MFA 506 (UFP); idem, 18/VI/2003, Bezerra, MFA 530 (UFP); idem, 15/VIII/2002, Bezerra, MFA 644 (UFP).

8. Physarum bogoriense Racib., Hedwigia 37: 52. 1898.

Frutificação média, um único espécime constituído por 13 plasmodiocarpos, desenvolvidos em câmaraúmida sobre folha senescente de palmeira (Arecaceae), 
coletada no Cerrado (Mangabeira, $210 \mathrm{~m}$ alt.) durante a estação chuvosa (abril) e esporulados após 53 dias de cultivo.

Distribuição no Brasil: Região Nordeste (Bahia, Paraíba, Pernambuco, Piauí), Sudeste (São Paulo), Sul (Santa Catarina).

Material examinado: BRASIL. Sergipe: Areia Branca, Parque Nacional Serra de Itabaiana, 28/V/2002, Bezerra, MFA 961 (UFP).

9. Physarum cinereum (Batsch) Pers., Neues Mag. Bot. 1: 89. 1794.

Frutificações abundantes, > 50 esporângios, esporulados sobre fezes de coelho brasileiro, após 33 dias em câmara-úmida e também coletados no campo durante a estação seca (setembro, dezembro, fevereiro e março), sobre folhas de dicotiledôneas não identificadas, procedentes do Cerrado (Mangabeira, $210 \mathrm{~m}$ alt.) e da mata de galeria (Riacho Coqueiro, $180 \mathrm{~m}$ alt.).

Distribuição no Brasil: Região Norte (Amazonas), Nordeste (Bahia, Pernambuco), Sudeste (Minas Gerais, São Paulo), Sul (Santa Catarina).

Material examinado: BRASIL. Sergipe: Areia Branca, Parque Nacional Serra de Itabaiana, 12/II/2003, Bezerra, MFA 146 (UFP); idem, 13/II/2003, Bezerra, MFA 147 (UFP); idem, 13/II/2003, Bezerra, MFA 305 (UFP); idem, 13/III/2003, Bezerra, MFA 315 (UFP); idem, 13/III/2003, Bezerra, MFA 316 (UFP); idem, 13/III/2003, Bezerra, MFA 317 (UFP); idem, 13/III/2003, Bezerra, MFA 318 (UFP); idem, 13/III/2003, Bezerra, MFA 319 (UFP); idem, 13/III/2003, Bezerra, MFA 321 (UFP); idem, 20/IX/2002, Bezerra, MFA 934 (UFP); idem, 4/XII/2002, Bezerra, MFA 1113 (UFP).

10. Physarum compressum Alb. \& Schwein., Consp. Fung. Lusat: 97 (1805).

Frutificação média, um único espécime constituído por 12 esporângios pedicelados, esporulados após sete meses de cultivo em câmara-úmida sobre folha morta não identificada, coletada no Cerrado (Mangabeira, $210 \mathrm{~m}$ alt.), durante a estação seca (dezembro).

Distribuição no Brasil: Região Nordeste (Bahia, Ceará, Maranhão, Pernambuco), Sudeste (São Paulo), Sul (Paraná, Rio Grande do Sul, Santa Catarina).

Material examinado: BRASIL. Sergipe: Areia Branca, Parque Nacional Serra de Itabaiana, 12/XII/2002, Bezerra, MFA 821 (UFP).

11. Physarum aff. conglomeratum (Fr.) Rostaf., Sluzowce Monogr. 108 (1874).

Fig. 1c-1d

Frutificação média, constituída por dois conjuntos de esporocarpos densamente agrupados, 15 a 24 esporângios sésseis, pulvinado-depressos, angulosos por compressão mútua, 0,5-1,0 mm no seu maior diâmetro, 0,1-0,2 $\mathrm{mm}$ alt. Esporoteca amarelo-esverdeado, iridescente, com concentrações de cálcio amareloalaranjado no centro (99.s.g.y, Anonymous 1976); hipotalo não visualizado; perídio simples, deiscência apical e irregular, amarelo (83. brill. Y, Anonymous 1976) por luz transmitida; capilício hialino, liso, com nódulos de cálcio amarelo-ouro brilhante; esporada castanho-escuro, esporo castanho-oliváceo (8.g y. Pink, Anonymous 1976) por luz transmitida, liso (imersão), $6 \mu \mathrm{m}$ diâm.

O material estudado foi coletado em mata de galeria (Riacho Coqueiro, $180 \mathrm{~m}$ alt.), sobre folha de dicotiledônea não identificada, durante a estação chuvosa (junho). Difere de quase todas as espécies com esporângios sésseis descritas para o gênero, assemelhando-se a Physarum conglomeratum (Fr.) Rostaf., encontrado no folhedo de florestas da Europa, Índia e América do Norte (Martin \& Alexopoulos 1969), mas não incluído para os Neotrópicos por Farr (1976) nem nas listagens feitas recentemente para o Brasil (Cavalcanti 2002; Maimoni-Rodella 2002; Putzke 2002).

Os esporocarpos possuem perídio simples e esporos de dimensão menor que a referida para $P$. conglomeratum. Poderia se tratar de uma nova espécie para a Ciência porém, segundo o pensamento atual (Clark 2004; Stephenson et al. 2004), novas amostras devem ser obtidas para confirmar que se trata efetivamente de um novo táxon e não de uma variação morfotípica ou biotípica de $P$. conglomeratum.

Distribuição no Brasil: com registro apenas em Areia Branca, Sergipe.

Material examinado: BRASIL. Sergipe: Areia Branca, Parque Nacional Serra de Itabaiana, 17/VI/2003, Bezerra, MFA 469 (UFP; MA-Fungi).

12. Physarum crateriforme Petch, Ann. Roy. Bot. Gard. (Peradeniya) 4: 304 (1909).

Frutificação abundante, um único espécime constituído por 22 esporângios pedicelados, muito típicos, coletado sobre casca do tronco vivo de Moraceae (Artocarpus heterophyllus Lam.) no Sítio Noel, vertente Oeste (180 m alt.), durante a estiagem (setembro).

Distribuição no Brasil: Região Nordeste (Pernambuco).

Material examinado: BRASIL. Sergipe: Areia Branca, Parque Nacional Serra de Itabaiana, 10/IX/2003, Bezerra, MFA 839 (UFP; MA-Fungi).

13. Physarum decipiens M.A. Curtis, Amer. J. Sci. Arts 6: 352 (1849).

Frutificação abundante, um único espécime constituído por 20 plasmodiocarpos e alguns esporângios sésseis, esporulados em câmara-úmida após 43 dias de 
cultivo sobre folhas de palmeiras, coletadas no Cerrado (Mangabeira, $210 \mathrm{~m}$ ), durante a estiagem (janeiro).

Distribuição no Brasil: Região Nordeste (Pernambuco), Região Sudeste (São Paulo).

Material examinado: BRASIL. Sergipe: Areia Branca, Parque Nacional Serra de Itabaiana, 19/I/2003, Bezerra, MFA 1022 (UFP).

14. Physarum leucophaeum Fr., Symb. Gasteromyc.: 24 (1818).

Frutificação desde escassa até abundante, $>50$ esporângios pedicelados, coletados no campo sobre tronco morto caído e apenas 1 esporocarpo esporulado em câmara-úmida montada com Cactaceae (Melocactus sp.), após 15 dias de cultivo. Material procedente do topo da Serra $660 \mathrm{~m}$, assim como a $427 \mathrm{~m}$ alt. e $210 \mathrm{~m}$ alt. e no Cerrado (Mangabeira), durante a estação chuvosa (maio) e a estiagem (fevereiro e setembro).

Distribuição no Brasil: Região Nordeste (Pernambuco), Sudeste (São Paulo).

Material examinado: BRASIL. Sergipe: Areia Branca, Parque Nacional Serra de Itabaiana, 14/II/2003, Bezerra, MFA 382 (UFP); idem, 15/V/2003, Bezerra, MFA 609 (UFP); idem, 1/IX/2002, Bezerra, MFA 1003 (UFP).

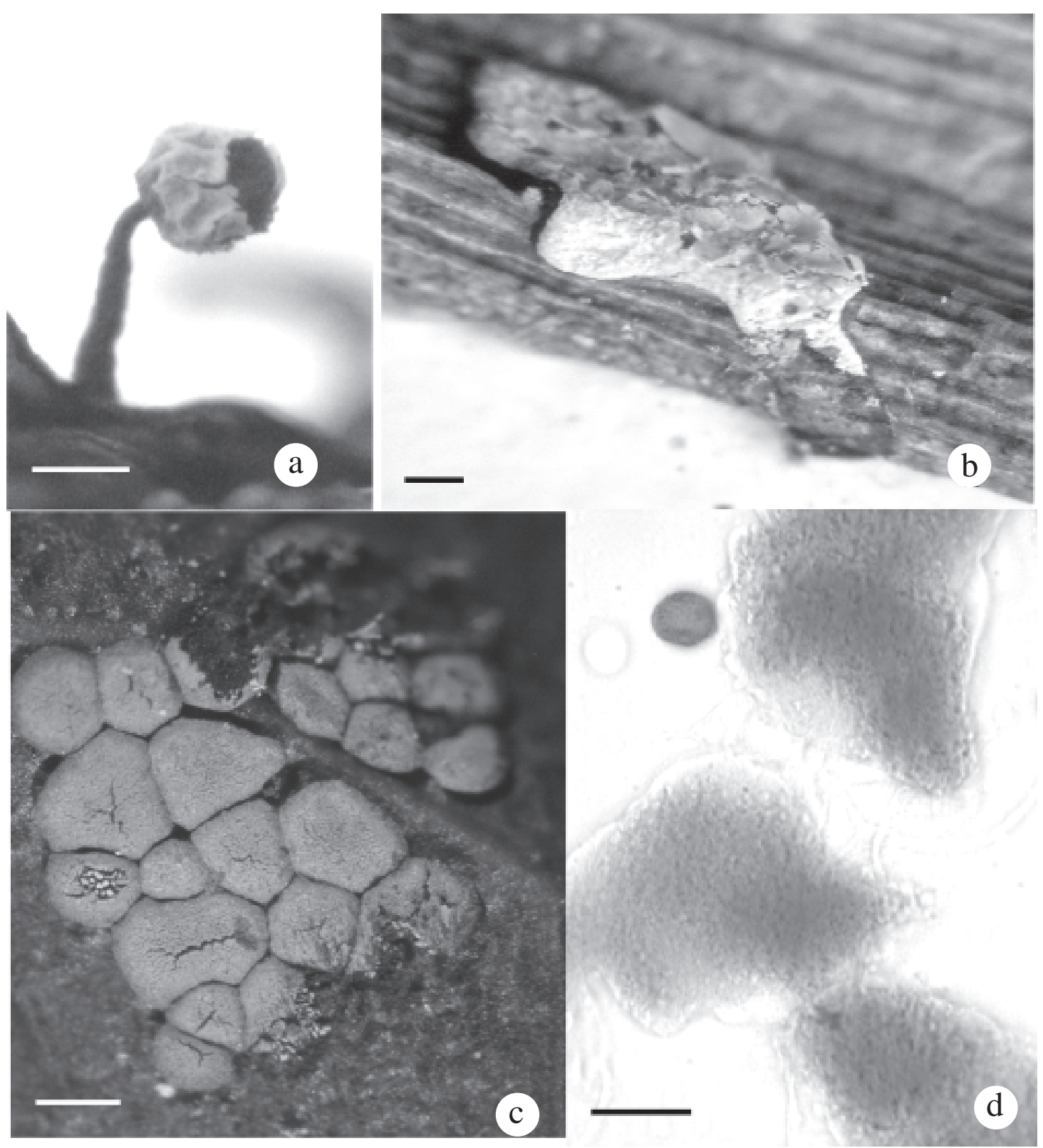

Figura 1. Esporocarpos de Diderma rugosum (Rex) T. Macbr., Didymium dubium Rostaf. e Physarum aff. conglomeratum (Fr.) Rostaf. procedentes do Parque Nacional Serra de Itabaiana, SE, Brasil. a. Esporângio evidenciando a típica deiscência do perídio. b. Plasmodiocarpo. c. Esporângios sésseis densamente agrupados, sobre folha. d. Nódulos calcários, filamentos capiliciais e esporos em microscopia óptica. Barras: $\mathrm{a}=0,2 \mathrm{~mm}, \mathrm{~b}=0,1 \mathrm{~mm}, \mathrm{c}=0,4 \mathrm{~mm}, \mathrm{~d}=10 \mu \mathrm{m}$. 
15. Physarum melleum (Berk. \& Broome) Massee, Monogr. Myxogastr.: 278 (1892).

Frutificações abundantes, 65-100 esporângios pedicelados, sobre folhas mortas ou senescentes de Bromeliaceae coletadas na Serra (427 m alt.) durante a estiagem (dezembro).

Distribuição no Brasil: Região Norte (Amazonas), Nordeste (Bahia, Pernambuco, Piauí), Sudeste (São Paulo), Sul (Paraná, Santa Catarina).

Material examinado: BRASIL. Sergipe: Areia Branca, Parque Nacional Serra de Itabaiana, 21/XII/2003, Bezerra, MFA 680 (UFP); idem, 21/XII/2003, Bezerra, MFA 685 (UFP; MA-Fungi); idem, 21/XII/2003, Bezerra, MFA 691 (UFP).

16. Physarum nucleatum Rex, Proc. Acad. Nat. Sci. Philadelphia 43: 389 (1891).

Frutificações de médias até abundantes, 17 a >50 esporângios pedicelados, todos sobre troncos mortos caídos, em mata de galeria (Riacho Coqueiro, $180 \mathrm{~m}$ alt.) e na trilha de acesso ao Salão do Rio dos Negros (210 m alt.), durante a estação chuvosa (maio, agosto) e na estiagem (setembro, outubro).

Distribuição no Brasil: Região Norte (Amazonas, Roraima), Nordeste (Ceará, Pernambuco, Piauí), Sudeste (São Paulo), Sul (Paraná).

Material examinado: BRASIL. Sergipe: Areia Branca, Parque Nacional Serra de Itabaiana, 14/V/2002, Bezerra, MFA 103 (UFP); idem, 14/VIII/2002, Bezerra, MFA 157 (UFP); idem, 16/VIII/2002, Bezerra, MFA 165 (UFP); idem, 12/IX/2002, Bezerra, MFA 459 (UFP); idem, 14/X/2003, Bezerra, MFA 730 (UFP).

17. Physarum oblatum T. Macbr., Bull. Iowa Univ. Lab. Nat. Hist. 2: 384 (1893).

Frutificações abundantes, $>50$ esporângios, sobre tronco morto caído de pau-pombo (Tapirira guianensis Aubl., Anacardiaceae) e de maria-farinha (não identificada), coletadas na vertente Leste a $427 \mathrm{~m}$ alt. e no Sítio Noel, vertente Oeste (210 m alt.), durante a estação chuvosa (maio, agosto).

Distribuição no Brasil: Região Nordeste (Bahia, Paraíba, Pernambuco).

Material examinado: BRASIL. Sergipe: Areia Branca, Parque Nacional Serra de Itabaiana, 15/V/2003, Bezerra, MFA 608 (UFP); idem, 14/VIII/2003, Bezerra, MFA $728 b$ (UFP).

18. Physarum penetrale Rex, Proc. Acad. Nat. Sci. Philadelphia 43: 389 (1891).

Frutificações abundantes, 30 a mais de 50 esporângios pedicelados, muito típicos, coletados sobre troncos mortos caídos, em mata de galeria (Riacho Coqueiro, $180 \mathrm{~m}$ alt.), durante a estiagem (setembro).

Distribuição no Brasil: Região Nordeste (Pernambuco), Sudeste (São Paulo).

Material examinado: BRASIL. Sergipe: Areia Branca, Estação Ecológica Serra de Itabaiana, 12/IX/2003, Bezerra, MFA 871 (UFP); idem, 12/IX/2003, Bezerra, MFA 875 (UFP).

19. Physarum pulcherrimum Berk. \& Ravenel in Berkeley, Grevillea 2: 65(1873).

Fig. 2a-b

Frutificações de médias até abundantes, 13 a mais de 50 esporângios pedicelados, sobre folhas mortas e troncos mortos caídos, coletados em mata de galeria (Riacho Coqueiro, $180 \mathrm{~m}$ alt.), na trilha de acesso ao Salão do Rio dos Negros ( $210 \mathrm{~m}$ alt.) e no topo da Serra (670 m alt.), durante a estação chuvosa (abril, junho, julho, agosto).

Esta espécie foi referida por Farr (1976) para os Neotrópicos com base em um espécime procedente do Paraguai, coletado no século XIX e até o momento não se dispunha de qualquer referência para o Brasil. Existe grande semelhança com Physarum roseum Berk. \& Broome, do qual difere pelo tamanho dos nódulos de cálcio do capilício e características da columela. O material coletado no PNSI apresenta esporângios pedicelados, globosos a subglobosos, esporoteca de coloração vermelho-violáceo (11. v. Red) e pedicelo de um vermelho mais escuro (13. deep Red). No material coletado nas diferentes localidades do PNSI os esporos apresentam diâmetro menor que o referido para a espécie $(6 \mu \mathrm{m})$ e não foi observada a presença de columela, mas esta pode estar ocasionalmente ausente, como descrevem Martin \& Alexopoulos (1969).

Distribuição no Brasil: primeiro registro para o país.

Material examinado: BRASIL. Sergipe: Areia Branca, Parque Nacional Serra de Itabaiana, 15/VII/2002, Bezerra, MFA 136 (UFP; MA-Fungi); idem, 11/IV/2003, Bezerra, MFA 24la, (UFP); idem, 18/VI/2003, Bezerra, MFA 505 (UFP; MA-Fungi); idem, 13/VIII/2003, Bezerra, MFA $709 b$ (UFP; MA-Fungi).

20. Physarum pusillum (Berk. \& M.A. Curtis) G. Lister in Lister, Monogr. Mycetozoa, ed. 2: 64 (1911).

Frutificações de médias até abundantes, 16 a >30 esporângios pedicelados, sobre troncos mortos caídos de mariafarinha (não identificada) e folhas mortas de Arecaceae e Bromeliaceae, coletadas em Cerrado (Mangabeira, $210 \mathrm{~m}$ alt.) e no topo da Serra (670 m alt.), durante a estiagem (fevereiro, dezembro) e na estação chuvosa (agosto).

Distribuição no Brasil: Região Nordeste (Bahia, Paraíba, Pernambuco, Piauí), Sudeste (São Paulo). 
Material examinado: BRASIL. Sergipe: Areia Branca, Parque Nacional Serra de Itabaiana, 13/VIII/2002, Bezerra, MFA 169 (UFP); idem, 14/II/2003, Bezerra, MFA 381 (UFP); idem, 13/VIII/2002, Bezerra, MFA 636 (UFP); idem, 21/XII/2003, Bezerra, MFA 677 (UFP); idem, 21/XII/2003, Bezerra, MFA 681 a (UFP).

21. Physarum roseum Berk. \& Broome, J. Linn. Soc., Bot. 14: 84 (1873).

Frutificação abundante, um único espécime constituído por 100 esporângios pedicelados, originados de plasmódio vermelho desenvolvido após 94 dias de cultivo sobre fezes de coelho brasileiro, coletadas em Cerrado (Mangabeira, 180 m alt.), durante a estação chuvosa (abril).

Distribuição no Brasil: Região Nordeste (Pernambuco), Sudeste (São Paulo).

Material examinado: BRASIL. Sergipe: Areia Branca, Parque Nacional Serra de Itabaiana, 22/IV/2003, Bezerra, MFA 932 (UFP).

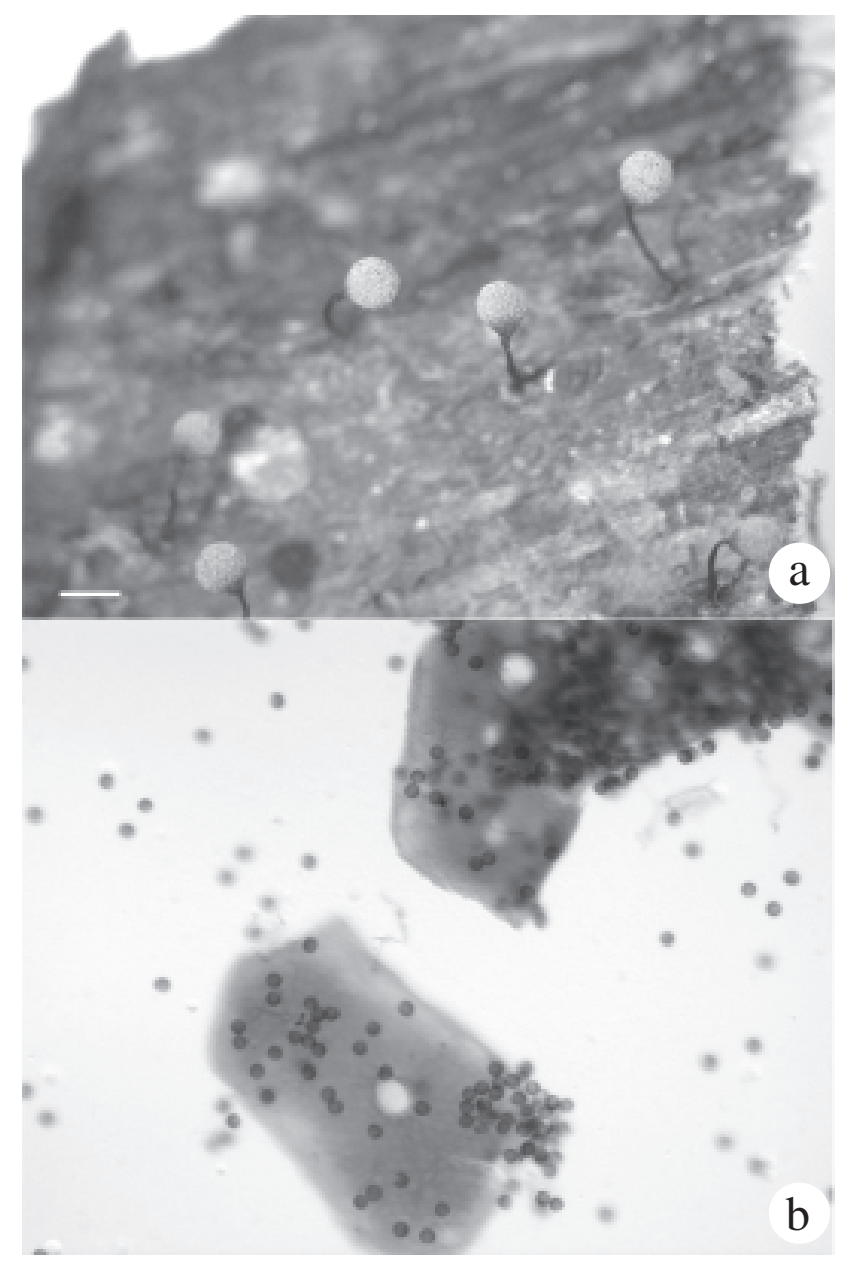

Figura 2. Esporocarpos de Physarum pulcherrimum Berk. procedente do Parque Nacional Serra de Itabaiana, SE, Brasil. a. Esporângios sobre tronco morto. b. Nódulos calcários, capilício e esporos em microscopia óptica. Barras: $\mathrm{a}=0,5 \mathrm{~mm}, \mathrm{~b}=50 \mu \mathrm{m}$.
22. Physarum stellatum (Massee) G.W. Martin, Mycologia 39(4): 461(1947).

Frutificações abundantes, $>50$ esporângios pedicelados, todos sobre troncos mortos, em pé ou caídos, de dicotiledôneas não identificadas. Coletas efetuadas em mata de galeria (Riacho Coqueiro, $180 \mathrm{~m}$ alt.), Gruta da Serra (220 m alt.), trilha de acesso ao Salão do Rio dos Negros (210 m alt.), e no topo da Serra (660 m alt.), durante a estação chuvosa (julho) e na estiagem (setembro, novembro).

Distribuição no Brasil: Região Norte (Amazonas, Amapá), Nordeste (Bahia, Ceará, Pernambuco, Piauí), Centro-Oeste (Distrito Federal), Sudeste (Rio de Janeiro, São Paulo), Sul (Paraná).

Material examinado: BRASIL. Sergipe: Areia Branca, Parque Nacional Serra de Itabaiana, 13/VII/2003, Bezerra, MFA 145 (UFP); idem, 13/VII/2002, Bezerra, MFA 339 (UFP); idem, 13/VII/2002, Bezerra, MFA 340 (UFP); 27/XI/2003, Bezerra, MFA $778 b$ (UFP); idem, 9/IX/2003, Bezerra, MFA 824 (UFP); idem, 9/IX/2003, Bezerra, MFA 825 (UFP); idem, 9/IX/2003, Bezerra, MFA 826 (UFP); idem, 9/IX/2003, Bezerra, MFA 828 (UFP); idem, 9/IX/2003, Bezerra, MFA 830 (UFP); idem, 9/IX/2003, Bezerra, MFA 831 (UFP); idem, 11/IX/2003, Bezerra, MFA 862a (UFP); idem, 27/XI/2003, Bezerra, MFA 1052 (UFP); idem, 29/XI/2003, Bezerra, MFA 1057 (UFP).

23. Physarum tenerum Rex, Proc. Acad. Nat. Sci. Philadelphia 42: 192(1890).

Frutificação abundante, $>50$ esporângios, sobre tronco morto caído, coletados na Gruta da Serra, (220 m alt.), durante a estação chuvosa (junho).

Distribuição no Brasil: Região Norte (Amazonas), Nordeste (Bahia, Pernambuco), Sudeste (São Paulo), Sul (Rio Grande do Sul).

Material examinado: BRASIL. Sergipe: Areia Branca, Parque Nacional Serra de Itabaiana, 19/VI/2003, Bezerra, MFA 558 (UFP).

24. Physarum viride (Bull.) Pers., Ann. Bot. (Usteri) 15: 6(1795).

Frutificações muito abundantes, 50 a $>100$ esporângios pedicelados, sobre troncos mortos em pé ou caídos, alguns com briófitas. Coletados em diferentes ambientes e altitudes: vertente Oeste, na localidade Bula Cinza (220 m alt.); vertente Leste, no alojamento do IBAMA, em mata de galeria (Riacho Coqueiro, $180 \mathrm{~m}$ alt.), na Gruta da Serra (220 m alt.) e no topo da Serra (657 $\mathrm{m}$ alt.). Esporula durante a estiagem (setembro, novembro, dezembro) e na estação chuvosa (abril, julho, agosto). 
Distribuição no Brasil: Região Norte (Amazonas), Nordeste (Pernambuco, Piauí), Sudeste (Rio de Janeiro, São Paulo), Sul (Paraná, Santa Catarina).

Material examinado: BRASIL. Sergipe: Areia Branca, Parque Nacional Serra de Itabaiana, 4/IV/2002, Bezerra, MFA 79 (UFP); idem, 16/VIII/2002, Bezerra, MFA 120 (UFP); idem, 13/VII/2002, Bezerra, MFA 159 (UFP); idem, 13/VII/2002, Bezerra, MFA 160 (UFP); idem, 11/IX/2002, Bezerra, MFA 432 (UFP); idem, 15/V/2003, Bezerra, MFA 620 (UFP); idem, 19/XII/2003, Bezerra, MFA 660 (UFP); idem, 21/XII/2003, Bezerra, MFA 697 a (UFP); idem, 13/VIII/2003, Bezerra, MFA 705 (UFP); idem, 28/XI/2003, Bezerra, MFA 792 (UFP).

Riqueza, abundância e distribuição das espécies de Physarales nos microhabitats analisados - Estão representados na mixobiota do PNSI 50\% dos gêneros de Physarales reconhecidos por Martin et al. (1983) e $80 \%$ dos que possuem registro de ocorrência no Brasil. As novas referências efetuadas no PNSI elevam para 82 o número de representantes de Physarales de ocorrência conhecida para o país. Analisando as listagens de Cavalcanti (2002), Maimoni-Rodella (2002) e Putzke (2002), verifica-se que, considerando somente as Physarales, a mixobiota do PNSI reúne mais espécies que o total conhecido para as Regiões Norte (42 spp., 18 Physarales) e Sul (78 spp., 31 Physarales) do país.

As Physaraceae predominam entre os exemplares da ordem obtidos nos diferentes ambientes e microhabitats analisados no PNSI (89,6\% das coleções), particularmente espécies de Physarum (72\%). Estão representados cinco dos seus 11 gêneros, correspondendo a $44 \%$ das espécies desta família de ocorrência conhecida até o momento para o Brasil.

As oito espécies ocorrentes na localidade Mangabeira (cerrado) não se incluem entre as 13 registradas no Riacho Coqueiro (mata de galeria), exceto $P$. cinereum, o que resulta em um coeficiente de comunidade muito baixo $(0,09)$ entre as mixobiotas dos dois ambientes. Em estudo efetuado em ambientes de mata úmida e cerrado, no município de Botucatu, São Paulo, Maimoni-Rodella \& Gottsberger (1980) incluem Physaraceae entre as famílias mais freqüentes, tanto no cerrado, com oito espécies, quanto na mata úmida, com sete espécies; Didymiaceae, representada apenas por D. clavus e D. minus (Lister) Morgan, foi colocada como a terceira família com maior freqüência no cerrado, não sendo registrada na área de mata úmida estudada pelos referidos autores. As Didymiaceae também são pouco representadas nos ambientes de mata e cerrado analisados no PNSI, ocorrendo Diachea silvaepluvialis e Diderma deplanatum na mata de galeria e Didymium dubium no cerrado. No estudo efetuado por Cavalcanti (1978) sobre a ocorrência de mixomicetos corticícolas em áreas de cerradão e cerrado, no Estado de São Paulo, as duas famílias estão representadas, correspondendo a $25 \%$ do total encontrado; também predominam as Physaraceae, com uma espécie de Badhamia e cinco de Physarum e três espécies são comuns às registradas no cerrado do PNSI: P. album, P. bogoriense e P. tenerum.

Ao efetuarem estudos no Parque Nacional de Sete Cidades, Piauí, em ambiente de mata ciliar e cerrado, Mobin \& Cavalcanti (1999) e Cavalcanti \& Mobin (2004) registraram a presença de 47 espécies de mixomicetos, das quais 21 representam as Physarales, com predominância das Physaraceae (17 spp.) sobre as Didymiaceae (4 spp.); todas as espécies comportaramse como folíícolas, esporulando sobre folhas mortas das palmeiras Astrocaryum vulgare Mart., Copernicia prunifera (Miller) H.E. Moore e Mauritia flexuosa L.f.; embora ocupando distintos microhabitats, quatro gêneros e nove espécies são comuns à mixobiota do PNSI: D. clavus, $P$. oblonga, C. leucocephalum, C. paraguayense, P. album, $P$. melleum, $P$. nucleatum, $P$. stellatum e $P$. viride.

Novozhilov et al. (2001) comentam que a disponibilidade de biótopos e microhabitats influencia a distribuição dos Myxomycetes e consideram que os quatro principais tipos possuem diferentes grupos ecológicos a eles adaptados. Segundo estes autores, no maior grupo, dos lignícolas, ocorrem 30-70\% das espécies que compõem a Classe, grande parte delas com esporocarpos macroscópicos, relativamente fáceis de serem visualizados no campo, com picos de esporulação bem definidos; os corticícolas reúnem 20-40\% ou mesmo até $80 \%$ das espécies nos ambientes áridos, quase todas com esporocarpos diminutos, necessitando do cultivo em câmara-úmida para serem detectadas; as foliícolas reúnem 20-70\% das espécies, principalmente Physarales, de esporocarpos macroscópicos porém difíceis de serem visualizados no folhedo, também necessitando da técnica de câmara-úmida; o quarto tipo reúne as fimícolas, pequeno grupo de espécies que habitam fezes de herbívoros, mais comuns em ambientes áridos, encontradas no campo porém melhor percebidas em câmara-úmida, após um período longo de cultivo. Todos estes tipos de microhabitats são ocupados pelas Physarales no PNSI, ainda ocorrendo, embora menos freqüentes e diversificadas, as suculentícolas e as muscícolas (Tab. 1).

As câmaras-úmidas possibilitaram o registro de menos espécies (6 spp.) do que as coletas de campo (27 spp.), embora as fimícolas e suculentícolas tenham se desenvolvido exclusivamente em cultivo de laboratório. Apenas P. leucophaeum e P. cinereum tiveram registros tanto em coletas de campo quanto em cultivo de câmara-úmida. 
Tabela 1. Distribuição das espécies de Physarales nos diferentes grupos ecológicos, de acordo com os substratos de esporulação em que foram coletados os espécimes no Parque Nacional Serra de Itabaiana, SE, Brasil.

\begin{tabular}{|c|c|}
\hline Grupo ecológico & Espécies \\
\hline Corticícola & Craterium aureum (Schumach.) Rostaf. Physarum crateriforme Petch. \\
\hline Fimícola & Physarum cinereum (Batsch) Pers. Physarum roseum Berk. \& Broome. \\
\hline Foliícola & $\begin{array}{l}\text { Craterium leucocephalum (Pers. ex J.F. Gmel.) Ditmar. Craterium paraguayense (Speg.) Lister. Diachea } \\
\text { silvaepluvialis M.L. Farr. Diderma deplanatum Fr. Didymium dubium Rostaf. Physarum bogoriense Racib. } \\
\text { Physarum cinereum (Batsch) Pers.Physarum compressum Alb. \& Schwein. Physarum aff. conglomeratum } \\
\text { (Bull.) Chevall. Physarum decipiens M. A. Curtis. Physarum pulcherrimum Berk. \& Ravenel. Physarum } \\
\text { pusillum (Berk. \& M.A. Curtis) G. Lister. }\end{array}$ \\
\hline Lignícola & $\begin{array}{l}\text { Badhamia sp. Craterium paraguayense (Speg.) Lister. Didymium clavus (Alb. \& Schwein.) Rabenh. Fuligo } \\
\text { septica (L.) F.H. Wigg. Physarella oblonga (Berk. \& M.A. Curtis) Morgan. Physarum album (Bull.) Chevall. } \\
\text { Physarum leucophaeum Fr. Physarum nucleatum Rex. Physarum oblatum T. Macbr. Physarum penetrale } \\
\text { Rex. Physarum pulcherrimum Berk. \& Ravenel. Physarum pusillum (Berk. \& M.A.Curtis) G. Lister. Physarum } \\
\text { stellatum (Massee) G.W. Martin. Physarum tenerum Rex. Physarum viride (Bull.) Pers. }\end{array}$ \\
\hline Muscícola & Craterium aureum (Schumach.) Rostaf. Diderma rugosum (Rex) T. Macbr. Diderma sp. \\
\hline Suculentícola & $\begin{array}{l}\text { Diderma effusum (Schwein.) Morgan. Didymium clavus (Alb. \& Schwein.) Rabenh. Craterium leucocephalum } \\
\text { (Pers. ex J.F. Gmel.) Ditmar. Craterium paraguayense (Speg.) Lister. Physarum leucophaeum Fr. Physarum } \\
\text { melleum (Berk. \& Broome) Massee. Physarum pusillum (Berk. \& M.A. Curtis) G. Lister. }\end{array}$ \\
\hline
\end{tabular}

Ogata et al. (1996) comentam que combinações de fatores climáticos, como chuvas torrenciais e umidade ambiente elevada, associados à presença de um grande número de insetos e aracnídeos que se alimentam dos esporocarpos, excessiva acidez do substrato e fracas correntes de ar nas densas florestas tropicais onde a entrada de luz é reduzida, influenciam a estacionalidade, abundância e freqüência das espécies. Chiappeta et al. (2003), em estudos realizados em Jaboatão dos Guararapes, no Estado de Pernambuco, também observaram a relação entre a abundância de plasmodiocarpos de Fuligo septica e a quantidade de chuvas ocorridas em meses anteriores à esporulação. No presente estudo, a riqueza de espécies parece não ter sido afetada pela pluviosidade, uma vez que um número semelhante de gêneros e espécies de Physarales foram encontrados na estação chuvosa ( 5 gên., 15 spp.) e na estiagem ( 7 gên.,19 spp.), e oito espécies, pertencentes a três diferentes gêneros, ocorreram nas duas estações do ano (Tab. 2); o mesmo se observa quanto à abundância, com igual número de espécies escassas ocorrendo nas duas estações. O grupo das lignícolas, com seis gêneros e 15 espécies, é o mais rico na mixobiota do PNSI, esporulando na estiagem e na estação chuvosa e reunindo as espécies mais abundantes, como $P$. viride e $P$. stellatum. Seguem-se as foliícolas, com cinco gêneros e 13 espécies, presentes nas duas estações do ano; neste grupo ocorre a maior parte das Didymiaceae. Considerando os microhabitats mais especializados, os pequenos grupos das corticícolas e muscícolas restringiram-se a ambientes mais úmidos, situados em diferentes altitudes, enquanto as fimícolas só foram encontradas no ambiente mais seco do Cerrado. No grupo das suculentícolas, registrado apenas em câmaras-úmidas montadas com substratos coletados na estiagem, estão representados dois gêneros de Didymiaceae e dois de Physaraceae.

Em estudos efetuados por Ogata et al. (1996) em Floresta Tropical Subperenifólia no México, onde ocorrem oito espécies de Physarales, $P$. viride foi incluída entre as dominantes, apesar de menos abundante que o observado em florestas temperadas e tropicais por Stephenson (1988) e Maimoni-Rodella \& Gottsberger (1980). Esta espécie também se inclui entre as Physarales mais abundantes nas diferentes localidades e altitudes estudadas no PNSI, assim como $P$. cinereum e P. stellatum (Tab. 2). Considerando a constância de ocorrência na mixobiota do PNSI, as espécies de Physarales são quase todas acidentais, mesmo as que se mostraram abundantes, como $P$. cinereum e $P$. stellatum. Foram enquadradas como acessórias apenas $P$. viride e P. oblonga, registradas durante a estação chuvosa e na estiagem, a primeira delas sendo encontrada em diferentes altitudes e localidades, nas duas vertentes da Serra. 
Tabela 2. Abundância, constância e distribuição estacional das espécies de Physarales ocorrentes no Parque Nacional Serra de Itabaiana, SE, Brasil. Abundância: escassa (<1,5\%); ocasional (1,5-3,5\%); comum ( $>3,5-6,5 \%)$; abundante $(>6,5 \%)$. Constância: acidental $(<25 \%)$; acessória 25-50\%; constante $>50 \%$. Chuvosa: março-agosto. Estiagem: setembro-fevereiro.

\begin{tabular}{|c|c|c|c|}
\hline Espécie & Abundância & Constância & Estação do ano \\
\hline Diachea silvaepluvialis M.L. Farr & escassa & acidental & estiagem \\
\hline D. deplanatum Fr. & escassa & acidental & estiagem \\
\hline D. effusum (Schwein.) Morgan & escassa & acidental & estiagem \\
\hline D. rugosum $(\mathrm{Rex}) \mathrm{T}$. Macbr. & ocasional & acidental & estiagem \\
\hline Diderma sp. & escassa & acidental & chuvosa \\
\hline Didymium clavus (Alb. \& Schwein.) Rabenh. & comum & acidental & estiagem \\
\hline Badhamia sp. & escassa & acidental & chuvosa \\
\hline Craterium aureum ( Schumach.) Rostaf. & escassa & acidental & chuvosa \\
\hline C. leucocephalum (Pers. ex. J.F. Gmel.) Ditmar & comum & acidental & estiagem \\
\hline C. paraguayense (Speg.) Lister & comum & acidental & estiagem \\
\hline Fuligo septica (L.) F.H. Wigg. & escassa & acidental & estiagem \\
\hline Physarella oblonga (Berk. \& M.A. Curtis) Morgan & comum & acessória & estiagem/chuvosa \\
\hline Physarum album (Bull.) Chevall. & ocasional & acidental & chuvosa \\
\hline P. cinereum (Batsch) Pers. & abundante & acidental & estiagem \\
\hline P. aff. conglomeratum (Fr.) Rostaf. & escassa & acidental & chuvosa \\
\hline P. crateriforme Petch & escassa & acidental & estiagem \\
\hline P. leucophaeum Fr. & escassa & acidental & estiagem/chuvosa \\
\hline P. melleum (Berk. \& Broome) Massee & ocasional & acidental & estiagem \\
\hline P. nucleatum Rex & ocasional & acidental & estiagem/chuvosa \\
\hline P. oblatum T. Macbr. & escassa & acidental & chuvosa \\
\hline P. penetrale Rex & escassa & acidental & estiagem \\
\hline P. pulcherrimum Berk. \& Ravenel & ocasional & acidental & estiagem/chuvosa \\
\hline P. pusillum (Berk. \& M.A. Curtis) G. Lister & ocasional & acidental & estiagem/chuvosa \\
\hline P. stellatum (Massee) G.W. Martin & abundante & acidental & estiagem/chuvosa \\
\hline P. tenerum Rex & escassa & acidental & chuvosa \\
\hline P. viride (Bull.) Pers. & abundante & acessória & estiagem/chuvosa \\
\hline
\end{tabular}

\section{Agradecimentos}

Os autores agradecem à direção do Parque Nacional Serra de Itabaiana, pelas facilidades concedidas durante o período de coleta, particularmente à Valdineide Santana e Marleno Costa, pelo apoio logístico durante o período de trabalhos de campo; agradecem ainda o apoio financeiro propiciado pelo $\mathrm{CNPq}$, pela concessão de bolsas a L.H. Cavalcanti (Proc. 303392/2003-7; 490087/2003-5) e M.F.A. Bezerra (Proc. 141157/2002-0).

\section{Referências bibliográficas}

Anonymous. 1976. ISCC-NBS color-name charts ilustrated with centroid colors. Inter. Society Color Council, National Bureau of Standars, Washington, DC, USA.

Cavalcanti, L.H. 1978. Mixomicetos do Cerrado II. Análise comparativa das espécies encontradas no Cerrado protegido e no queimado anualmente. Pp. 129-140. In: Anais do 28 Congresso Nacional de Botânica. Belo Horizonte, MG. (CNB1977).

Cavalcanti, L.H. 2002. Biodiversidade e distribuição de mixomicetos em ambientes naturais e antropogênicos no Brasil: espécies ocorrentes nas Regiões Norte e Nordeste. Pp. 209-216. In: E.L. Araujo; A.N. Moura; E.V.S.B. Sampaio; L.M. Gestinari \& J.M.T. Carneiro. (eds.). Biodiversidade, conservação e uso sustentável da flora do Brasil. Recife, Universidade Federal Rural de Pernambuco, Sociedade Botânica do Brasil.
Cavalcanti, L.H. \& Mobin, M. 2004. Myxomycetes associated with palm trees at the Sete Cidades National Park, Piauí State, Brazil. Systematics and Geography of Plants 74 (1): 109-127.

Chiappeta, A.A.; Sena, K.X.R. \& Cavalcanti, L.H. 2003. Evironmental factors affecting sporulation of Fuligo septica (Myxomycetes) on sugar cane bagasse. Brazilian Archives of Biology and Tecnology 46:7-12.

Clark, J. 2004. Reproductive systems and taxonomy in the myxomycetes. Systematics and Geography of Plants 74: 209-216.

CONDESE. 1978. Projeto básico para a implantação da Estação Ecológica Serra de Itabaiana-SE. Aracajú.

Eliasson, U.H. \& Keller, H.W. 1999. Coprofilous Myxomycetes: updated summary, key to species, and taxonomic observation on Trichia brunnea, Arcyria elaterensis and Arcyria stipata. Karstenia 39: 1-10.

Estrada-Torres, A.; Lado, C. \& Rodríguez-Palma, M. 2001. Two new species of Myxomycetes from a tropical deciduous forest of Mexico. Mycologia 93: 744-750.

Farr, M.L. 1976. Flora Neotropica. New York, Organization for Flora Neotropica. New York, New York Botanical Garden.

Hagelstein, R. 1944. The Mycetozoa of North America. New York, Hafner.

Härkönen, M. \& Vänskä, H. 2004. Corticolous myxomycetes and lichens in the Botanical Garden in Helsinki, Finland: a comparison after decades of recovering from air pollution. Systematics and Geography of Plants 74: 183-187.

Lado, C. 2001. Nomenmyx. A nomenclatural taxabase of Myxomycetes. Cuadernos de Trabajo Flora Micológica Ibérica 16. Consejo Superior de Investigaciones Científicas. Madrid, Real Jardín Botánico, CSIC. 
Lado, C. 2004. Nivicolous myxomycetes of the Iberian Peninsula: considerations on species richness and ecological requirements. Systematics and Geography of Plants 74: 143-157.

Lado, C.; Ronikier, A.; Ronikier, M. \& Drozdowicz, A. 2005. Nivicolous Myxomycetes from the Sierra de Gredos (Central Spain). Nova Hedwigia 81: 371-394.

Lakhanpal, T.N. \& Mukerji, K.G. 1981. Taxonomy of the Indian Myxomycetes. Bibl. Mycol. Bd 78, A. Vaduz: A.R. Gantner Verlag K.G.

Lister, A. 1925. A Monograph of the Mycetozoa. $2^{\text {nd }}$. ed. London, Britsh Museum.

Macbride, T. \& Martin, G.W. 1934. The Myxomycetes. New York, MacMillan Co.

Maimoni-Rodella, R.C. 2002. Biodiversidade e distribuição de mixomicetos em ambientes naturais e antropogênicos no Brasil: espécies ocorrentes nas Regiões Sudeste e Centro-Oeste. Pp.217-220 In: E.L. Araujo; A.N. Moura; E.V.S.B. Sampaio; L.M. Gestinari \& J.M.T. Carneiro (eds.). Biodiversidade, conservação e uso sustentável da flora do Brasil. Recife, Universidade Federal Rural de Pernambuco, Sociedade Botânica do Brasil.

Maimoni-Rodella, R.C. \& Gottsberger, G. 1980. Myxomycetes from the forest and the cerrado vegetation in Botucatu, Brazil: A comparative ecological study. Nova Hedwigia 34: 204-247.

Martin, G.W. \& Alexopoulos, C.J. 1969. The Myxomycetes. Iowa City, University of Iowa Press.

Martin, G.W.; Alexopoulos, C.J. \& Farr, M.L. 1983. The Genera of Myxomycetes. Iowa City, University of Iowa Press.

Mitchell, D. 2004. A key to corticolous Myxomycota. Systematics and Geography of Plants 74: 261-285.

Mobin, M. \& Cavalcanti, L.H. 1999. Physarales (Myxomycetes) do Parque Nacional de Sete Cidades (Piauí, Brasil). Hoehnea 26: $1-14$.
Nannenga-Bremekamp, N.E. 1991. A Guide to temperate Myxomycetes. Bristol, Biopress Limited.

Novozhilov, Y.K.; Schnittler, M.; Rollins, A.W. \& Stephenson, S.L. 2001. Myxomycetes from different forest types in Puerto Rico. Mycotaxon 77: 285-299.

Ogata, N.; Rico-Grey, V. \& Nestel, D. 1996. Abundance, richness, and diversity of Myxomycetes in a Neotropical Forest Ravine. Biotropica 28: 627-635.

Putzke, J. 2002. Myxomycetes na Região Sul do Brasil. Pp. 221-223. In: E.L. Araujo; A.N. Moura; E.V.S.B. Sampaio; L.M. Gestinari \& J.M.T. Carneiro (eds.). Biodiversidade, conservação e uso sustentável da flora do Brasil. Recife, Universidade Federal Rural de Pernambuco, Sociedade Botânica do Brasil.

Schnittler, M.; Lado, C. \& Stephenson, S.L. 2002. Rapid biodivesity assessment on a tropical myxomycete assemblage-Maquipucuna Cloud Forest Reserve. Ecuador. Fungal Diversity 9: 135-167.

Schnittler, M. \& Stephenson, S.L. 2002. Inflorescences of Neotropical herbs as a newly discovered microhabitat for myxomycetes. Mycologia 94: 6-20.

Stephenson, S.L. 1988. Distribution and ecology of Myxomycetes in temperate forests. 1. Patterns of occurrence in the upland forests of Southwestern Virginia. Canadian Journal of Botany 66: 2187-2207.

Stephenson, S.L.; Landolf, J.C. \& Moore, D.L. 1999. Prostostelids, dictyostelids and Myxomycetes in the litter microhabitat of the Luquillo Experimental Forest, Puerto Rico. Mycological Research 103: 209-214.

Stephenson, S.L.; Schnittler, M.; Lado, C.; Estrada-Torres, A.; Basanta, D.W.; Landolt, J.C.; Novozhilov, Y.K.; Clark, J.; Moore, D.L. \& Spiegel, F.W. 2004. Studies of neotropical mycetozoans. Systematics and Geography of Plants 74: 87-108.

Wingley-Basanta, D. 2004. The effect of simulated acid rain on corticolous myxomycetes. Systematics and Geography of Plants 74: 175-181. 